\title{
A SNP based linkage map of the turkey genome reveals multiple intrachromosomal rearrangements between the Turkey and Chicken genomes
}

\author{
Muhammad L Aslam*, John WM Bastiaansen', Richard PMA Crooijmans' ${ }^{1}$, Addie Vereijken², \\ Hendrik-Jan Megens', Martien AM Groenen ${ }^{1}$
}

\begin{abstract}
Background: The turkey (Meleagris gallopavo) is an important agricultural species that is the second largest contributor to the world's poultry meat production. The genomic resources of turkey provide turkey breeders with tools needed for the genetic improvement of commercial breeds of turkey for economically important traits. A linkage map of turkey is essential not only for the mapping of quantitative trait loci, but also as a framework to enable the assignment of sequence contigs to specific chromosomes. Comparative genomics with chicken provides insight into mechanisms of genome evolution and helps in identifying rare genomic events such as genomic rearrangements and duplications/deletions.

Results: Eighteen full sib families, comprising 1008 (35 F1 and 973 F2) birds, were genotyped for 775 single nucleotide polymorphisms (SNPs). Of the 775 SNPs, 570 were informative and used to construct a linkage map in turkey. The final map contains 531 markers in 28 linkage groups. The total genetic distance covered by these linkage groups is 2,324 centimorgans (CM) with the largest linkage group (81 loci) measuring $326 \mathrm{cM}$. Average marker interval for all markers across the 28 linkage groups is $4.6 \mathrm{cM}$. Comparative mapping of turkey and chicken revealed two inter-, and 57 intrachromosomal rearrangements between these two species.
\end{abstract}

Conclusion: Our turkey genetic map of 531 markers reveals a genome length of 2,324 cM. Our linkage map provides an improvement of previously published maps because of the more even distribution of the markers and because the map is completely based on SNP markers enabling easier and faster genotyping assays than the microsatellitemarkers used in previous linkage maps. Turkey and chicken are shown to have a highly conserved genomic structure with a relatively low number of inter-, and intrachromosomal rearrangements.

\section{Background}

The turkey (Meleagris gallopavo, MGA) is an important agricultural species that is largely used as a meat type bird. In 2008, turkey represented $6.65 \%$ of the world poultry meat production [1]. The world-wide turkey population has rapidly grown due to increased commercial farming. Global turkey stocks nearly tripled from 178 million in 1970 to over 482 million in 2008. Over the same time period, the production volume increased more than fivefold from 1.2 to 6.1 million tons [1].

\footnotetext{
* Correspondence: muhammadl.aslam@wur.nl

'Animal Breeding and Genomics Centre, Wageningen University,6709PG, Wageningen, The Netherlands

Full list of author information is available at the end of the article
}

The turkey genome consists of 39 pairs of autosomes and 1 pair of sex chromosomes [2]. The predicted size of the turkey genome is 1.1 billion bases on the turkey genome build UMD 2.01 which is based on sequences from a combination of two next generation sequencing platforms, Roche 454 and Illumina GAII with $5 \times$ and $25 \times$ coverage respectively. Limited information is available on the turkey linkage and physical map although a small number of low resolution linkage maps using microsatellite markers $[2,3]$ have been published. Linkage maps of chicken on the other hand are more abundant and have generally used larger numbers of markers [4-10]. Comparative cytogenetic and linkage maps between turkey and chicken showed conserved synteny and close ancestral relation among these species $[2,3]$

\section{() Biomed Central}


and support the hypothetical ancestral Galliform karyotype [11]. Chromosome banding and zoo-FISH with chromosome paints for the turkey and chicken chromosomes have suggested that chicken and turkey karyotypes are distinguished by at least two interchromosomal rearrangements $[2,12,13]$. Chicken chromosome 2 and 4 are represented by turkey chromosomes 3 and 6 and by turkey chromosomes 4 and 9 respectively $[2,3,13]$.

Chromosome studies have revealed that the karyotype is more conserved among avian lineages than it is among other groups, such as mammals, with most avian species showing a diploid chromosome number between 76 and 80 http://www.genomesize.com. This suggests that chromosomal evolution or large-scale rearrangements affecting chromosome number occur at a low rate in birds and as a result many chromosomes have remained more or less intact during avian evolution [14]. Chicken chromosome specific probes have been used for in situ hybridization onto metaphase spreads of other birds and revealed an overall picture of a high degree of chromosomal homology between chicken and representatives from many avian orders [15]. Hybridization results also indicated that interchromosomal rearrangements have been rare during avian evolution $[16,17]$.

A linkage map is essential for the mapping of quantitative trait loci (QTL) and very useful for the assembly of genome sequence and subsequently mapping of genes along the chromosomes. A high-resolution linkage map facilitates fine mapping of quantitative trait loci (QTLs) and can be produced because of the abundance of SNPs within the genome [18]. SNP based genotyping is preferred because it is highly accurate, quick and automated, using limited human intervention. Increasing the marker density of the linkage map further enables the analyses of genomic sequences associated with high recombination rates [9].

The present study was designed to develop a SNP based linkage map in turkey and to detect genomic rearrangements between turkey and chicken.

\section{Methods}

\section{Experimental population}

Parents were randomly selected from two different lines to produce F1 offspring. Ten parent males were randomly selected from a line that was selected for high growth and ten parent females were randomly selected from a line that was selected for high reproduction. Average body weight of males in the high growth line from which ten parent males were randomly selected was $20.6 \mathrm{Kg}$ at 20 weeks of age and the average egg production of females in high reproduction line from which ten parent females were randomly selected was 115.5 hatching eggs/24 weeks. An F2 generation of 18 full sib families was produced by crossing 17 randomly selected F1 males and 18 randomly selected F1 females. One male was mated with two females, other F1 parents were mated only once. In total, 973 F2 offspring were produced with an average full sib family size of 54.1 with a range from 31-90 individuals. All families were used for the SNPs genotyping to construct linkage maps of different chromosomes.

\section{DNA isolation}

Genomic DNA was isolated from blood samples collected in 10\% EDTA using either the automated nucleic acid extraction CAS-1820 X-tractor Gene (Corbett Life Science), or the manual nucleic acid extraction using Gentra Puregene Blood Kit (Qiagen) following manufacturer's protocol with minor modifications.

DNA concentrations were measured using ND-1000 Spectrophotometer (NanoDrop) and diluted to the required concentration of $50 \eta \mathrm{g} \mu \mathrm{L}^{-1}$.

\section{SNP selection}

Previously, we identified 11,287 SNPs in turkey by sequencing reduced representation libraries on an Illumina GA sequencer [19]. To achieve an even spacing of SNPs across the 40 turkey chromosomes while a turkey genome sequence was not available, SNPs in turkey were selected based on their orthologous position on the chicken genome sequence (WASHUC2 build, May 2006). Currently the chicken genome [20] covers 30 of the 39 chromosomes in chicken which comprises approximately $95 \%$ of chicken genome. By this approach, we did not select SNPs in parts of the turkey genome that are syntenic to genomic regions in chicken that are currently not represented in the chicken genome assembly. Assembled turkey short read contigs from Kerstens et al. [19] that contained SNPs were mapped on the chicken genome. Short read contigs in the size range of 50-100 bp were mapped using Megablast [21] and short read contigs of $100 \mathrm{bp}$ and longer were mapped using BlastZ [22] with contig alignment criteria of at least $80 \%$ alignment and at least $60 \%$ sequence identity. In total 6,537 SNPs could be assigned a syntenic location on the chicken genome. In addition to chicken genome location, the final selection criteria for SNPs also included the Illumina design score and the estimated minor allele frequency based on the Illumina sequences from Kerstens et al [19]. The distance (in bps) between the selected SNPs was varied based on the size of the chromosome, because of the higher recombination frequency on the microchromosomes of birds. Chicken chromosomes were divided into three groups; $1-10+$ Z, 11-19 and 20-28 + LGE22 and the average SNP spacing chosen for these three groups was 
1.4-1.9 SNP per $\mathrm{Mb}, 0.7-1.0 \mathrm{SNP}$ per $\mathrm{Mb}$ and 0.4-0.6 SNP per Mb respectively.

In addition, seven SNPs derived from 5 different turkey genes i.e. Pit1, AFABP, PRKAG3, IGF2 and GDF8 were also used.

\section{Genotyping}

Two 384-plex GoldenGate oligo pool assay (OPA) sets were designed for genotyping using VeraCode technology on an Illumina BeadXpress Reader. The GoldenGate assay was performed according to manufacturer's protocol and as described in Fan et al. [23] and Hyten et al. [24]. Automated genotype clustering and calling was performed with GenomeStudio ${ }^{\mathrm{Tm}}$ data analysis software (Illumina). All genotype calling results were manually checked and any obvious errors in calling the homozygous or heterozygous clusters were corrected.

SNPs selected from the 5 turkey genes (Pit1, AFABP, PRKAG3, IGF2 and GDF8) were genotyped with an ABI SNaPshot assay and analyzed on an ABI 3730 DNA Analyzer (Additional file 1).

\section{Genetic Linkage analysis}

Genotyping data was filtered by removing uninformative markers, markers giving Mendelian errors in more than one families and markers with low call rate as described by Groenen et al. [9]. The modified CRI-MAP software version 2.4 [25] by Xuelu Liu (Monsanto), which can handle much larger numbers of markers segregating in complex pedigrees was utilized for the linkage analysis.

Map building was performed step by step using AUTOGROUP, BUILD, CHROMPIC, FLIPSN, and FIXED options of CRI-MAP according to the procedures used by Stapley et al. [26] and Elferink et al. [10]. Using AUTOGROUP, parameter layers utilized for getting linkage groups were as follows: layer $1(20,0,2,0.3)$; layer 2 $(20,0,20,0.3)$; layer $3(10,0,20,0.3)$ and layer $4(5,0,20$, $0.3)$. Layer 4 had minimum stringency with likelihood ratio (LOD score) $>5$, 0 times the average number of meiosis, shared linkages with not more than 20 groups and with 0.3 of minimum linkage ratio [25]. Linkage groups were assigned to specific turkey chromosomes using the already known physical positions of turkey SNPs in the chicken genome and comparative information from the cytogenetic study of Griffin et al. [2] on turkey and chicken. Turkey chromosome names were assigned using the nomenclature used by Griffin et al. [2].

Maps are reported as sex averaged maps unless otherwise indicated and map figures were drawn with the MapChart software version 2.2 [27].

\section{Comparative genetic analysis}

The order of SNPs on our linkage map was compared to the expected order based on the turkey and chicken genome assemblies UMD 2.01 and WASHUC2, respectively. Positions on the chicken genome were obtained earlier in the SNP selection step. Positions on the turkey genome were obtained by aligning SNP flanking sequences $\left(<1.0 \times \mathrm{E}^{-4}\right)$ using BLAST with megablast option [28] against the turkey reference genome sequence (UMD 2.01).

The turkey physical map order of SNPs was used to validate the linkage map order with CRI-MAP using the BUILD option. The order of SNPs in linkage maps was modified if the physical map order had a higher likelihood and total chromosome map length was smaller than the linkage map order. The genetic distance between the terminal markers of every chromosome from the turkey linkage map was compared to the genetic distance between the corresponding positions of the chicken genome. First, the sequence positions (bp) of these terminal turkey markers were found on the chicken physical map. Second, chicken markers were taken from the study of Elferink et al. [10] at the closest position (bp) to these sequence positions (bp). Finally the genetic distance between these chicken markers was calculated and compared to the turkey map length.

\section{Analysis of recombination rate and sequence motif densities}

The physical distance $(\mathrm{Mb})$ on turkey chromosomes was calculated between the first and the last SNP of the linkage map using the blastall option in BLAST [28]. Number of $\mathrm{Mb}$ covered by the linkage map (cM) was used to calculate recombination rate $(\mathrm{cM} / \mathrm{Mb})$ for every turkey chromosome which was compared to the physical size $(\mathrm{Mb})$ of the chromosomes [9,26]. The recombination rates $(\mathrm{cM} / \mathrm{Mb})$ were also compared to those for the chicken chromosomes described by Elferink et al. [10].

Densities of sequence motifs/elements CCCCCCC, CCTCCCT, CTCTCCC, CpG and CTCF consensus sequence CCNCCNGGNGG were found to vary with chromosome in chicken [9], therefore we also calculated these densities for each turkey chromosome from the turkey genome sequence (UMD 2.01). Only the part of the chromosome sequence covered by the linkage map was used to calculate these densities. Number of elements per $\mathrm{Mb}$ was calculated and compared against chromosome length (cM) except for CpG that was compared against $\mathrm{cM} / \mathrm{Mb}[9]$.

\section{Ethical approval for the use of animals in this study}

Although animals were used in this experimental work, no direct experiments were performed on them. Blood sample collection was carried out by licensed and authorized personnel under approval of Hendrix Genetics. No approval from the ethics committee was necessary. 


\section{Results}

\section{Genotyping results}

Genotyping call rates with an average of 0.80 were obtained. In total, 775 SNPs $(2 \times 384$-plex GoldenGate + 7 additional SNPs) were selected for genotyping and out of these, $98 \mathrm{SNP}$ assays failed (missing genotypes in the whole population), 80 SNPs appeared to be monomorphic (AA, or BB genotype) or positive for parologous sequences (all genotypes $\mathrm{AB}$ ), $13 \mathrm{SNPs}$ showed non-Mendelian inheritance in more than one family and 14 SNPs had zero informative meiosis. In total 205 SNPs were removed from the dataset.

\section{Linkage maps}

After filtering of genotyping data, 570 SNP markers were left for the linkage analysis. Of the total 570 markers that met all quality criteria, 531 markers were found significantly linked which were subsequently inserted at their most likely position (BUILD option, LOD $>3$ ) on one of 28 linkage groups that subsequently were assigned to 27 autosomes and the $\mathrm{Z}$ chromosome (Table 1). The number of informative meiosis for a marker varied from 7 to 666 with an average of 255 . The largest chromosome, MGA1, had a map with 81 SNPs and a map size of $325.8 \mathrm{cM}$, followed by MGA2 with 55 SNPs and a map size of $229 \mathrm{cM}$. The chromosomes MGA25 and MGA30 had the lowest number of SNPs (4 each) as well as the smallest map sizes with map lengths of 23.5 and $6.3 \mathrm{cM}$ respectively (Table 1 ). The total length of the sex average map (excluding the $\mathrm{Z}$ chromosome) was 2,165 cM and the average marker spacing was $4.4 \mathrm{cM}$. Sex specific analysis showed a difference in the male and the female maps. For $70 \%$ of chromosomes, male maps were longer than female maps, except for chromosomes MGA10, 11, 15, 16, 23, 25, 26 and MGA28 where the female maps were longer (Table 1). In general, a difference in length of $9 \%$ was observed between sex specific maps.

\section{Comparative genetic results Marker order}

For all except three of the turkey chromosomes the comparison of the linkage and the physical maps did not reveal any differences. For the three chromosomes, MGA2, 11 and 17, the marker order from the physical maps, showed a higher likelihood and a smaller map distance than the marker order obtained from our linkage analyses. Log likelihood values for MGA2, 11 and 17 were increased by 20.6, 98.6 and 0.7 and map distance reduced by $4.0,17.0$ and $1.3 \mathrm{cM}$ respectively. For these three chromosomes the marker order based on the physical map was used in further analyses.

Marker orders were found to be highly conserved between the turkey linkage and the chicken physical maps although 57 rearrangements were still detected between these species. The order of the SNP markers on chromosomes MGA14, 21, 25, 26 and MGAZ even showed $100 \%$ accordance with the order in the syntenic chicken chromosomes (Additional file 2).

The linkage maps for the turkey and the chicken chromosomes generally showed small differences in their lengths. Three exceptions are turkey chromosomes MGA1, MGA7 and MGAZ that showed a difference of more than $25 \mathrm{cM}$ with their syntenic chicken chromosomes GGA1 GGA7 and GGAZ. Whole genome genetic map size of chicken was $72.5 \mathrm{cM}$ larger than the whole genome genetic map size of turkey. In the comparisons of genetic lengths of turkey and chicken chromosomes, the difference in the reference genome positions (bp) of turkey SNPs genotyped in the present study and the genome positions (bp) of chicken SNPs used in the study by Elferink et al. [10] were small. On average the distance between the reference positions was 58,614 bp which will have caused an average difference of 0.28 $\mathrm{cM} /$ chromosome based on the average figure of $4.8 \mathrm{cM}$ per $\mathrm{Mb}$ in Turkey. The total physical map size of turkey covered by markers genotyped in this study was 939.4 $\mathrm{Mb}$. This is smaller than the region of the chicken physical map covered by the turkey genetic map which is 1146.5 Mb (Table 1).

\section{Rearrangements}

Two interchromosomal and 57 intrachromosomal rearrangements were observed between turkey and chicken (Figure 1). Two linkage groups, MGA3 and MGA6 were obtained from the SNPs selected with syntenic positions on chicken chromosome 2 and similarly two linkage groups, MGA4 and 9 were obtained from the SNPs selected from chicken chromosome 4 (Figure 1). These chromosomes (MGA3, 6 and MGA4, 9 Vs GGA2 and GGA3 respectively) did not only show interchromosomal rearrangements, but also showed multiple intrachromosomal rearrangements between turkey and chicken (Figure 1).

Regions with inverted marker order were observed on turkey chromosomes 10 and 20 when compared to their syntenic chicken chromosomes GGA8 and GGA18 (Figure 2). Other complex intrachromosomal rearrangements were also observed on turkey chromosome 1, 2, $5,8,11,12,13,15,16,17,19,22$, and 28 when compared to their syntenic chicken chromosomes (Additional Files $2 \& 3$ ).

The number of rearrangements per $\mathrm{Mb}$ varied considerably for different chromosomes. The average number of rearrangements per $\mathrm{Mb}$ for larger chromosomes (MGA1-MGA10) was 0.06, ranging from 0.01-0.13 with highest rate of rearrangements of 0.13 per $\mathrm{Mb}$ on MGA10. The average number of rearrangements per $\mathrm{Mb}$ for the smaller chromosomes (MGA11-MGA30) 
Table 1 Comparison of maps of turkey and chicken chromosomes based on genetic and physical sizes

\begin{tabular}{|c|c|c|c|c|c|c|c|c|}
\hline & & & Turkey & & & & Chicken & \\
\hline Chromosome & Number of SNPs & Female (cM) & Male (cM) & Average (cM) & Length (Mb) & Chromosome & $\begin{array}{c}\text { Genetic } \\
\text { length }(\mathrm{cM})\end{array}$ & $\begin{array}{c}\text { Syntenic } \\
\text { region }(\mathrm{Mb})\end{array}$ \\
\hline MGA1 & 81 & 318.9 & 344.7 & 325.8 & 200.7 & GGA1 & 353.4 & 193.3 \\
\hline MGA2 & 55 & 216.4 & 249.9 & 229 & 115.1 & GGA3 & 233.5 & 111.7 \\
\hline MGA3 & 40 & 140.6 & 149.5 & 140.9 & 89.7 & GGA2q & 149.5 & 134.7 \\
\hline MGA4 & 27 & 94 & 146.7 & 120.9 & 67.4 & GGA4q & 132.1 & 70.0 \\
\hline MGA5 & 33 & 108.1 & 131.3 & 118.3 & 59.8 & GGA5 & 116.2 & 61.2 \\
\hline MGA6 & 23 & 98.9 & 111.9 & 104.4 & 48.2 & GGA2p & 111.9 & 131.7 \\
\hline MGA7 & 22 & 65.3 & 77.3 & 71.4 & 29.6 & GGA7 & 102.5 & 37.5 \\
\hline MGA8 & 19 & 64.1 & 67.4 & 67.3 & 32.2 & GGA6 & 82.5 & 33.0 \\
\hline MGA9 & 10 & 52.9 & 59.7 & 55.2 & 16.8 & GGA4p & 60.5 & 91.3 \\
\hline MGA10 & 21 & 82.1 & 65.7 & 76.7 & 30.0 & GGA8 & 56.0 & 29.7 \\
\hline MGA11 & 19 & 64.1 & 54.6 & 59.8 & 22.8 & GGA9 & 78.0 & 22.3 \\
\hline MGA12 & 14 & 56.6 & 63.1 & 58.4 & 14.1 & GGA10 & 45.6 & 18.8 \\
\hline MGA13 & 17 & 51.2 & 59.8 & 54.4 & 18.0 & GGA11 & 62.9 & 20.9 \\
\hline MGA14 & 13 & 50.7 & 59.9 & 55.1 & 14.0 & GGA12 & 44.3 & 13.7 \\
\hline MGA15 & 21 & 59 & 56.9 & 59 & 16.1 & GGA13 & 56.2 & 17.4 \\
\hline MGA16 & 13 & 46.7 & 41.1 & 40.7 & 12.0 & GGA14 & 47.5 & 12.1 \\
\hline MGA17 & 13 & 57.5 & 59.5 & 57.5 & 12.5 & GGA15 & 52.7 & 12.3 \\
\hline MGA19 & 10 & 49.9 & 57.6 & 51.2 & 8.9 & GGA17 & 48.7 & 10.0 \\
\hline MGA20 & 12 & 54.6 & 67.3 & 60.6 & 9.3 & GGA18 & 48.7 & 9.3 \\
\hline MGA21 & 12 & 54.5 & 76 & 60.8 & 8.9 & GGA19 & 41.9 & 8.3 \\
\hline MGA22 & 10 & 53.8 & 60.3 & 56.2 & 11.3 & GGA20 & 42.0 & 10.6 \\
\hline MGA23 & 9 & 53.2 & 50.6 & 61.4 & 4.5 & GGA21 & 41.1 & 4.9 \\
\hline MGA24 & 4 & 26 & 38.6 & 33.1 & 1.9 & GGA22 & 21.6 & 1.8 \\
\hline MGA25 & 4 & 25.2 & 22.2 & 23.5 & 4.3 & GGA23 & 30.8 & 4.8 \\
\hline MGA26 & 8 & 71.3 & 50.7 & 57.3 & 6.0 & GGA24 & 51.8 & 5.7 \\
\hline MGA28 & 8 & 60.2 & 45 & 52.6 & 4.2 & GGA26 & 45.5 & 4.3 \\
\hline MGA30 & 4 & 1.4 & 4.9 & 6.3 & 1.1 & GGA28 & 17.1 & 0.9 \\
\hline Total autosomal & 522 & 2077.2 & 2272.2 & 2164.8 & 859.4 & Total & 2174.5 & 1072.2 \\
\hline MGAZ & 9 & - & 159.1 & 159.1 & 80.02 & GGAZ & 221.9 & 74.3 \\
\hline Total & 531 & 2077.2 & 2431.3 & 2323.9 & 939.4 & Total & 2396.4 & 1146.5 \\
\hline
\end{tabular}

was 0.11 , ranging from $0.08-0.42$ with highest rate of rearrangements of 0.42 per Mb on MGA12.

Comparative analysis of the turkey linkage, the turkey physical and the chicken physical maps showed discordance in the chromosomal allocation of 6 SNPs to these maps (Table 2). The turkey linkage and the chicken physical maps agreed with each other in the chromosomal allocation of these 6 SNPs while the turkey physical map disagreed. For example, according to the turkey linkage and chicken physical maps the SNP MGS3A000968 was assigned to MGA1 and GGA1 while this SNP was positioned on MGA8 in the turkey physical map (Table 2). Fourteen SNPs could not be assigned to any position on the turkey physical map while the allocation of these 14 SNPs to the turkey linkage map and the chicken physical map also agreed with each other (Table 2).

\section{Recombination rate and sequence elements}

Recombination rate of turkey chromosomes varied from 1.6 to $17.2 \mathrm{cM} / \mathrm{Mb}$. The physical length of chromosomes showed an inverse relation with recombination rate while $\mathrm{CpG} / \mathrm{Mb}$ density across the chromosome showed a direct relation. Turkey and chicken chromosomes of smaller sizes showed higher recombination rates than chromosomes with larger sizes (Figure 3A). CpG content showed increasing values with increasing recombination rate, i.e. higher CpG content in smaller chromosomes (Figure 3B). The frequency of sequence elements (CTCF, CCTCCCT, CTCTCCC and CCCCCCC) per Mb was found to be 


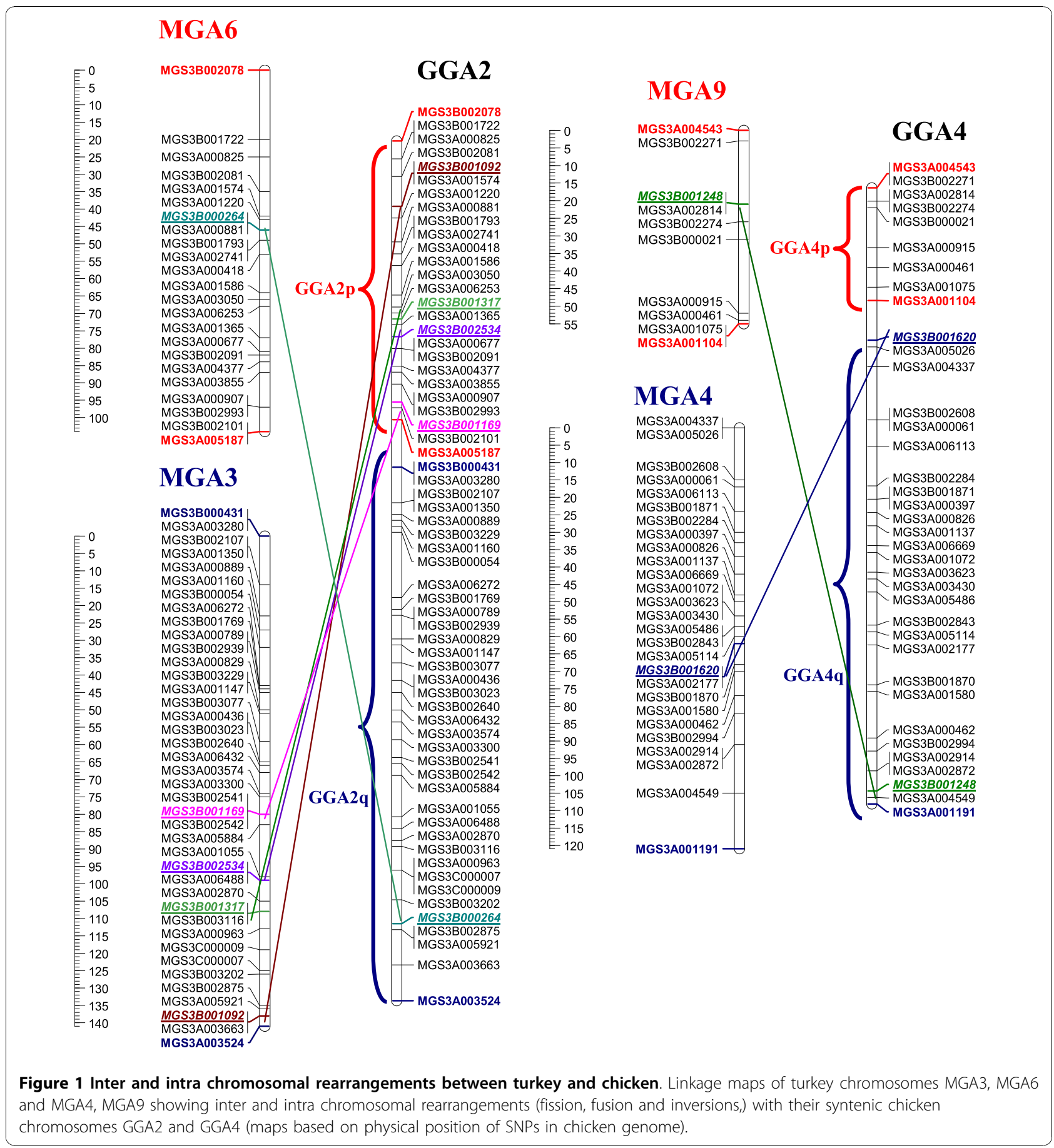

negatively correlated with the genetic size (cM) of chromosomes (Figure 3C-F).

\section{Discussion}

A whole genome SNP-based linkage map for the turkey is presented with 531 markers dispersed over 28 linkage groups and a total map length of $2324 \mathrm{cM}$. The total map length in the present study was slightly higher than that described by Reed et al. [3]. This difference in length is probably caused by coverage of three additional turkey chromosomes (MGA20, 24 and MGA25) along with the utilization of $69 \%$ higher number of markers in the present study, likely to be covering a larger proportion of the turkey genome. Matching the turkey linkage groups with the chicken physical map identified a map for each of the syntenic groups/chromosomes described 


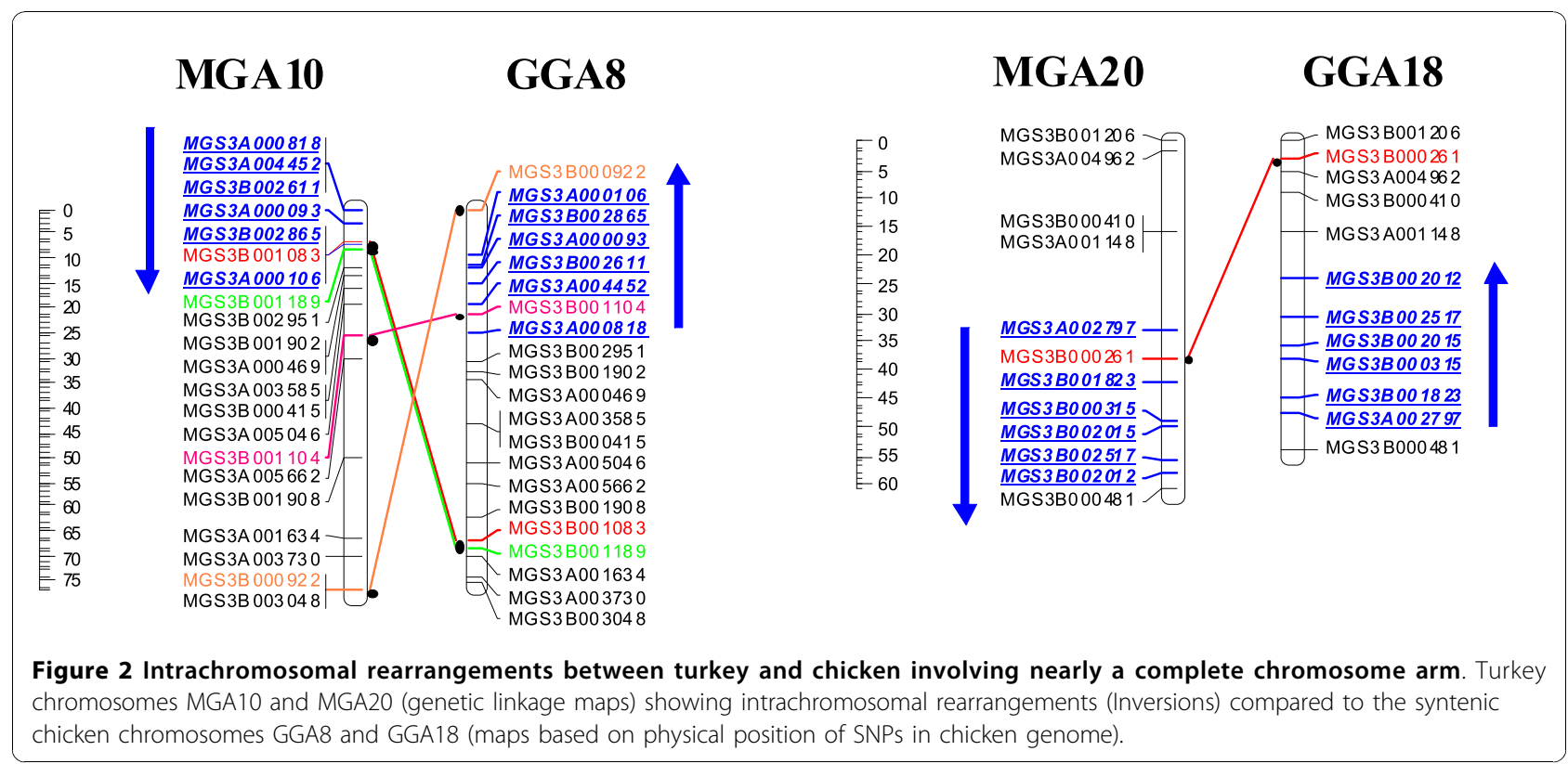

by Griffin et al. [2]. The comparison of the syntenic chromosomes between turkey and chicken showed that the genetic lengths of turkey chromosomes were very similar to the estimated genetic length of the chicken chromosomes (Table 1). When comparing the turkey genetic linkage map and chicken physical map with respect to the order of markers across the chromosomes, some of the chromosomes (MGA14, 21, 25, 26 and MGAZ) showed complete conservation in the order of markers whereas others showed limited variation. The conservation in the order of markers for the chicken chromosomes GGA12, 19, 24 and GGAZ with the syntenic turkey linkage groups was also observed by Reed et al. [3]. This high rate of concordance in the order of markers between the genomes of

Table 2 SNPs with discordance in allocation on turkey genome with turkey genetic and chicken physical map

\begin{tabular}{cccc}
\hline SNP_ID & Turkey Linkage map (MGA) & Turkey physical map (MGA) & Chicken physical map (GGA) \\
\hline MGS3A000968 & 1 & 8 & 1 \\
MGS3A003050 & 6 & 13 & 2 \\
MGS3A000053 & 8 & 19 & 6 \\
MGS3A004543 & 9 & 10 & 4 \\
MGS3A000578 & 15 & 14 & 13 \\
MGS3B002546 & 19 & 8 & 17 \\
MGS3A005799 & 1 & NA & 1 \\
MGS3B003240 & 2 & NA & 3 \\
MGS3A007335 & 2 & NA & 3 \\
MGS3A005026 & 4 & NA & \\
MGS3B000939 & 5 & NA & 4 \\
MGS3A007520 & 5 & NA & 5 \\
MGS3A006539 & 7 & NA & 5 \\
MGS3A007601 & 15 & NA & 7 \\
MGS3A007553 & 16 & NA & 13 \\
MGS3A002797 & 20 & NA & 14 \\
MGS3C000006 & 1 & NA & 18 \\
MGS3C000009 & 3 & NA & 1 \\
MGS3B001450 & $Z$ & NA & 2 \\
MGS3B002754 & $Z$ & NA & $Z$ \\
\hline
\end{tabular}




\section{$\triangle$ Turkey - Chicken - MGAZ}
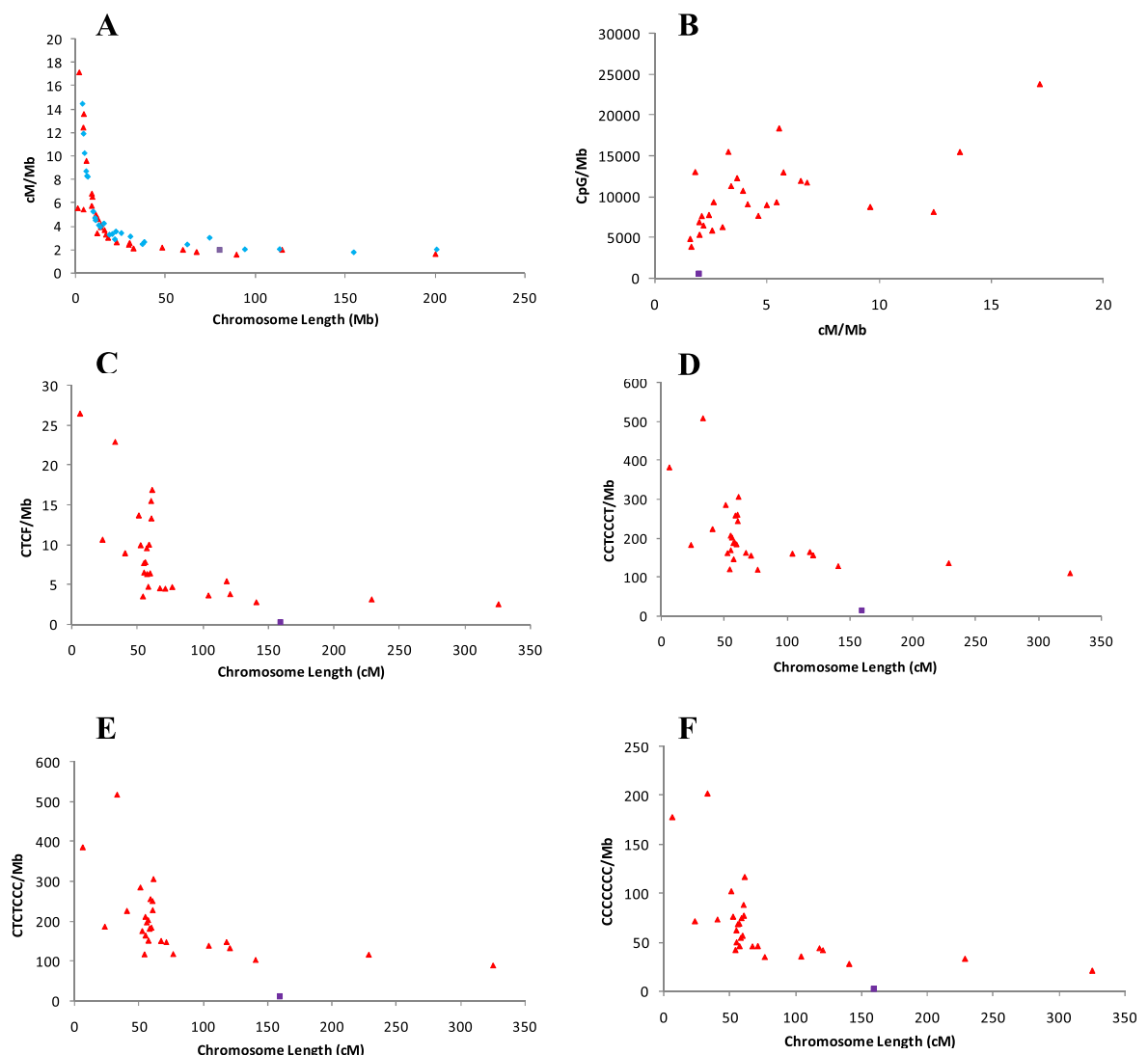

- = MGAZ had last marker in map with a high distance $(>30 \mathrm{cM})$ from the adjacent marker.

Figure 3 Distribution of recombination rates and sequence motif densities across the turkey chromosomes. Recombination rate $\mathrm{cM} / \mathrm{Mb}$ was correlated with chromosome length $(\mathrm{Mb})$ and $\mathrm{CpG} / \mathrm{Mb}$ was correlated with recombination rate (cM/Mb). All remaining sequence motifs (CCCCCCC, CCTCCCT, CTCF and CTCTCCC) were correlated with chromosome length (CM).

these two avian species is indicative of a highly conserved nature of avian genomes.

Observed interchromosomal rearrangements (Figure 1) in the present study between turkey and chicken are in agreement with the results of Griffin et al. [2]. A number of complex intrachromosomal rearrangements (inversions) were also observed between turkey and chicken. The observed large inverted regions, of nearly a complete chromosome arm on MGA10 and MGA20 in comparison to their syntenic chicken chromosome GGA8 and GGA18 (Figure 2) were also observed in a sequence based comparative study by Dalloul et al. [29]. Cytogenetic studies using chromosome painting also reported an inversion on MGA10 in comparison to the syntenic chicken chromosome GGA8 $[2,13]$. Our comparative linkage map of turkey and chicken does not show pericentic inversions on MGA2 and MGA3p as were reported by Griffin et al. [2] but we have observed complex rearrangements resulting in a reversed order of markers on these chromosomes (Additional file 3). Several other chromosomes, notably MGA1, 2, 5, 8, 11, 12, $13,15,16,17,19,22$, and MGA28 as well as the chromosomes that showed interchromosomal rearrangements (MGA3 and MGA6; MGA4 and MGA9) between turkey and chicken, also showed additional complex rearrangements probably involving multiple inversions or other complex rearrangements (Figure 1). A higher number of rearrangements per $\mathrm{Mb}$ were observed on the microchromosomes than on the macrochromosomes. The occurrence of this high number of rearrangements at the microchromosomes could be explained by the positive association of rearrangements with recombination rate [30].

Our observed low number of interchromosomal rearrangements between the chicken and turkey genomes, confirms previous results of a high degree of interchromosomal synteny in birds as seen within a number of different comparative studies of chicken with quail, duck 
and zebra finch [30-32]. It has been suggested that the low number of interchromosomal rearrangements during avian genome evolution is a consequence of the small amount of interspersed repeats, segmental duplications, and pseudogenes in avian genomes, which provide little opportunity for non allelic homozygous recombination $[33,34]$. A relatively high number of intrachromosomal rearrangements was observed in our comparative analysis of the turkey and chicken, which agrees with the findings of the sequence based comparative studies of chicken with turkey and zebra finch $[26,29,30]$. The relatively high number of intrachromosomal rearrangements clearly suggests that the organization of avian genomes is more prone to intrachromosomal rearrangements than previously appreciated based on chromosome banding and chromosome painting data [2].

The comparison of male vs. female maps showed differences in genetic lengths of maps. In turkey, the total malespecific map appeared to be $195 \mathrm{cM}$ longer than the female specific map. However, female-specific maps for some chromosomes (MGA10, 11, 15, 16, 23, 25, 26 and MGA28) were also found to be longer than the male maps (Table 1). The longer map length in homogametic males can be explained by the Haldane-Huxley rule $[35,36]$, which predicts that the frequency of recombination during meiosis is lower in the heterogametic sex. The smaller map lengths in turkey for some male-specific maps were found to be an exception to the Haldane-Huxley rule. However, the longer map lengths for some chromosome maps in the heterogametic sex were also found in chicken [9].

In the present study three maps i.e. the turkey genetic linkage map, the turkey physical map and the chicken physical map were compared. The discordance of turkey physical map with the turkey genetic linkage and the chicken physical map in the allocation of marker at different chromosomes could possibly be explained by the occurrence of assembly errors in the turkey genome sequence. The turkey physical map was created completely by whole genome shotgun sequencing using Roche 454 and Illumina GA2 sequence data. Inconsistencies between the turkey linkage and chicken physical maps relative to the turkey physical map are most likely a reflection of the challenge of correctly assembling a genome based on next-gen sequencing data alone. Markers that were in agreement between turkey linkage and chicken physical maps but that could not be positioned on the turkey physical map most likely reflect an uncovered genomic regions since the turkey genome sequence is known to cover around $95 \%$ of the complete genome (Turkey genome build UMD 2.01).

In general, higher recombination rates and higher densities of GC-rich elements were found on microchromosomes compared to macrochromosomes (Figure
3A \&3B). During meiosis, at least one chiasma per bivalent chromosome is required [37], but the likelihood of chiasmata forming varies along the chromosome [38]. In turkey, recombination rate and $\mathrm{GC}$ rich sequences (CTCF, CCTCCCT, CTCTCCC and CCCCCCC) were found to co-vary among different chromosomes. A similar trend was also seen in human, mouse and other birds like chicken and zebra finch $[9,10,20,26,37,39,40]$.

In the present study recombination rates were found to be correlated with $\mathrm{CpG} / \mathrm{Mb}$. In general $\mathrm{CpG} / \mathrm{Mb}$ tended to increase in areas of higher recombination i.e. microchromosomes (Figure 3B). This demonstrates that in the turkey microchromosomes, high recombination rate, high amount of GC-rich sequences (CTCF, CCTCCCT, CTCTCCC and CCCCCCC) and high amount of CpG contents are all correlated (Figure 3A-F). Other studies reported that $\mathrm{GC}$-rich regions in a genome had higher gene densities [41,42] and that microchromosomes had higher gene densities than the macrochromosomes [38]. The nature of the microchromosomes in birds, with their high recombination rates, high amount of $\mathrm{GC}$-rich sequences, GC content and gene densities appears to be an extreme instance of a general trend.

The results for MGAZ in the analysis of recombination rate and sequence motif densities across the chromosomes, were unexpected and MGAZ appeared as outlier as seen in figure 3A-F. This outlier spot could represent a true characteristic of MGAZ but more likely results from the low marker density on this particular chromosome in our analysis. (Additional Files 2 \&3).

\section{Conclusion}

Our SNP-based genetic linkage map of turkey with 531 markers reveals a genome length of 2,324 cM. This linkage map also allowed a comparison of the genome structures of turkey and chicken, demonstrating a very high degree of conservation in chromosome structure. A relatively low number of inter-, and intrachromosomal rearrangements was observed despite these two species being separated by 40 million years of evolution.

\section{Additional material}

Additional file 1: Detail of SBE primers along with their primer sequences and gene accession numbers. This file contains PCR reverse and forward primer sequences along with the SNP specific SBE primer sequence. This file also contains gene name and their accession numbers.

Additional file 2: Linkage and physical maps (data) of turkey chromosomes along with the physical map of syntenic chicken chromosomes. The detail of turkey linkage and physical maps along with the chicken physical map. This file also contains the flanking sequences of SNPS used in the present studied with their genotyping status. 
Additional file 3: Linkage maps (Figures) of turkey chromosomes showing rearrangements with syntenic chicken chromosomes Figures showing comparative linkage maps of turkey and chicken including all the chromosomes mentioned in the present paper.

\section{Acknowledgements}

The authors would like to thank Bert W Dibbits and Albertine Veenendaa (Animal Breeding and Genomics Centre, Wageningen University) for their help on the lab work. We also thank Gerard Albers and the Breeding Research and Technology Centre at Hendrix Genetics, for their help in the design of the experiment as well as the collection of blood samples.

\section{Author details}

${ }^{1}$ Animal Breeding and Genomics Centre, Wageningen University,6709PG, Wageningen, The Netherlands. ${ }^{2}$ Hendrix Genetics, Research \& Technology Centre, 5830 AC, Boxmeer, The Netherlands.

\section{Authors' contributions}

MLA and MAMG analyzed the data. RPMAC organized the lab work and improved the paper with suggestions and comments. MLA wrote the paper and all other authors gave suggestions and comments for the improvement of paper. All authors read and approved the final manuscript.

Received: 16 July 2010 Accepted: 20 November 2010 Published: 20 November 2010

\section{References}

1. Food and agriculture organization statistical division (FAOSTAT) of the United Nations. [http://faostat.fao.org/].

2. Griffin D, Robertson L, Tempest H, Vignal A, Fillon V, Crooijmans R, Groenen M, Deryusheva S, Gaginskaya E, Carre W, Waddington D, Talbot R, Volker M, Masabanda J, Burt D: Whole genome comparative studies between chicken and turkey and their implications for avian genome evolution. BMC Genomics 2008, 9(1):168

3. Reed KM, Chaves LD, Hall MK, Knutson TP, Harry DE: A comparative genetic map of the turkey genome. Cytogenet Genome Res 2005, 111:118-127.

4. Bumstead N, Palyga J: A preliminary linkage map of the chicken genome. Genomics 1992, 13(3):690-697.

5. Cheng HH, Levin I, Vallejo RL, Khatib H, Dodgson JB, Crittenden L, Hillel J: Development of a genetic map of the chicken with markers of high utility. Poult Sci 1995, 74:1855-1874.

6. Groenen MAM, Crooijmans RPMA, Veenendaal A, Cheng HH, Siwek M, Van der Poel JJ: A comprehensive microsatellite linkage map of the chicken genome. Genomics 1998, 49:265-274.

7. Herbergs J, Siwek M, Crooijmans RP, Van der Poel JJ, Groenen MA: Multicolour fluorescent detection and mapping of AFLP markers in chicken (Gallus domesticus). Anim Genet 1999, 30:274-285.

8. Groenen MAM, Cheng HH, Bumstead N, Benkel BF, Briles WE, Burke T, Burt DW, Crittenden LB, Dodgson J, Hillel J Lamont S, De Leon AP, Soller M, Takahashi $H$, Vignal A: A consensus linkage map of the chicken genome. Genome Res 2000, 10:137-147.

9. Groenen MAM, Wahlberg P, Foglio M, Cheng HH, Megens $\mathrm{H}-\mathrm{J}$, Crooijmans RPMA, Lathrop FBM, Muir WM, Ka-Shu Wong G, Gut I, Andersson L: A high-density SNP-based linkage map of the chicken genome reveals sequence features correlated with recombination rate. Genome Res 2009, 19(3):510-519.

10. Elferink M, van As P, Veenendaal T, Crooijmans R, Groenen M: Regional differences in recombination hotspots between two chicken populations. BMC Genetics 2010, 11(1):11.

11. Griffin DK, Robertson LBW, Tempest HG, Skinner BM: The evolution of the avian genome as revealed by molecular cytogenetics. Cytogenet Genome Res 2007, 117:64-77.

12. Stock AD, Bunch TD: The evolutionary implications of chromosomebanding pattern homologies in the bird order Galliformes. Cytogenet Cell Genet 1982, 34:136-148.

13. Shibusawa M, Nishibori M, Nishida-Umehara C, Tsudzuki M, Masabanda J, Griffin DK, Matsuda Y: Karyotypic evolution in the Galliformes: An examination of the process of karyotypic evolution by comparison of the molecular cytogenetic findings with the molecular phylogeny. Cytogenetic and Genome Research 2004, 106(1):111-119.

14. Ellegren $\mathrm{H}$ : Evolutionary stasis: the stable chromosomes of birds. Trends in Ecology \& Evolution 2010, 25(5):283-291.

15. Nie W, O'Brien PCM, Ng BL, Fu B, Volobouev V, Carter NP, FergusonSmith MA, Yang F: Avian comparative genomics: reciprocal chromosome painting between domestic chicken (Gallus gallus) and the stone curlew (Burhinus oedicnemus, Charadriiformes)-An atypical species with low diploid number. Chromosome Res 2009, 17(1):99-113.

16. Guttenbach M, Nanda I, Feichtinger W, Masabanda JS, Griffin DK, Schmid M: Comparative chromosome painting of chicken autosomal paints 1-9 in nine different bird species. Cytogenetic and Genome Research 2003, 103(12):173-184.

17. Derjusheva S, Kurganova A, Habermann F, Gaginskaya E: High chromosome conservation detected by comparative chromosome painting in chicken, pigeon and passerine birds. Chromosome Res 2004, 12:715-723.

18. Heil J, Glanowski S, Scott J, Winn-Deen E, McMullen I, Wu L, Gire C, Sprague A: An automated computer system to support ultra high throughput SNP genotyping. Pacific symposium on biocomputing: 2002 2002, 7:30-40.

19. Kerstens HHD, Crooijmans RPMA, Veenendaal A, Dibbits BW, Chin-AWoeng TFC, Dunnen JTd, Groenen MAM: Large scale single nucleotide polymorphism discovery in unsequenced genomes using second generation high throughput sequencing technology: applied to turkey. BMC Genomics 2009, 10:479.

20. Hillier LW, Miller W, Birney E, Warren W, Hardison RC, Ponting CP, Bork P, Burt DW, Groenen MAM, Delany ME, Dodgson JB, Chinwalla AT, Cliften PF, Clifton SW, Delehaunty KD, Fronick C, Fulton RS, Graves TA, Kremitzki C, Layman D, Magrini V, McPherson JD, Miner TL, Minx P, Nash WE, Nhan MN, Nelson JO, Oddy LG, Pohl CS, Randall-Maher J, et al: Sequence and comparative analysis of the chicken genome provide unique perspectives on vertebrate evolution. Nature 2004, 432:695-716.

21. Zhang Z, Schwartz S, Wagner L, Miller W: A greedy algorithm for aligning DNA sequences. J Comput Biol 2000, 7:203-214.

22. Schwartz S, Kent WJ, Smit A, Zhang Z, Baertsch R, Hardison RC, Haussler D, Miller W: Human-mouse alignments with BLASTZ. Genome Res 2003, 13:103-107.

23. Fan JB, Oliphant A, Shen R, Kermani BG, Garcia F, Gunderson KL, Hansen $M$, Steemers F, Butler SL, Deloukas P, Galver L, Hunt S, McBride C, Bibikova M, Rubano T, Chen J, Wickham E, Doucet D, Chang W, Campbell D, Zhang B, Kruglyak S, Bentley D, Haas J, Rigault P, Zhou L, Stuelpnagel J, Chee MS: Highly parallel SNP genotyping. Cold Spring Harb Symp Quant Biol 2003, 68:69-78.

24. Hyten DL, Song Q, Choi I-Y, Yoon M-S, Specht JE, Matukumalli LK, Nelson RL, Shoemaker RC, Young ND, Cregan PB: High-throughput genotyping with the GoldenGate assay in the complex genome of soybean. Theor Appl Genet 2008, 116:945-952.

25. Green P, Falls K, Crooks S: CRI-MAP Program. St. Louis: Washington university school of medicine, 2.41990.

26. Stapley J, Birkhead TR, Burke T, Slate J: A linkage map of the Zebra Finch Taeniopygia Guttata provides new insights into avian genome evolution. Genetics 2008, 179:651-667.

27. Voorrips RE: MapChart: Software for the graphical presentation of linkage maps and QTLs. J Hered 2002, 93(1):77-78.

28. Korf I, Yandell M, Bedell J: BLAST. O'Reilly Media 2003.

29. Dalloul RA, Long JA, Zimin AV, Aslam L, Beal K, Ann Blomberg L, Bouffard P, Burt DW, Crasta O, Crooijmans RPMA, Cooper K, Coulombe RA, De Supriyo, Delany ME, Dodgson JB, Dong JJ, Evans C, Frederickson KM, Flicek P, Florea L, Folkerts O, Groenen MAM, Harkins TT, Herrero J, Hoffmann S, Megens $\mathrm{H}$-Jan, Jiang A, de Jong P, Kaiser P, Kim H, et al: Multi-Platform Next-Generation Sequencing of the Domestic Turkey (Meleagris gallopavo): Genome Assembly and Analysis. PLoS Biol 2010, 8(9):e1000475.

30. Völker M, Backström N, Skinner BM, Langley EJ, Bunzey SK, Ellegren H, Griffin DK: Copy number variation, chromosome rearrangement, and their association with recombination during avian evolution. Genome Research 2010, 20(4):503-511.

31. Kayang $B$, Fillon $V$, Inoue-Murayama M, Miwa M, Leroux S, Feve $K$, Monvoisin J-L, Pitel F, Vignoles M, Mouilhayrat C, Beaumont C, Ito S, Minvielle F, Vignal A: Integrated maps in quail (Coturnix japonica) 
confirm the high degree of synteny conservation with chicken (Gallus gallus) despite 35 million years of divergence. BMC Genomics 2006, 7(1):101.

32. Skinner BM, Robertson LBW, Tempest HG, Langley EJ, loannou D, Fowler KE, Crooijmans RPMA, Hall AD, Griffin DK, Völker M: Comparative genomics in chicken and Pekin duck using FISH mapping and microarray analysis. BMC Genomics 2009, 10:357.

33. Burt DW, Bruley C, Dunn IC, Jones CT, Ramage A, Law AS, Morrice DR, Paton IR, Smith J, Windsor D, Sazanov A, Fries R, Waddington D: The dynamics of chromosome evolution in birds and mammals. Nature 1999, 402(6760):411-413.

34. Burt DW: Origin and evolution of avian microchromosomes. Cytogenetic and Genome Research 2002, 96(1-4):97-112.

35. Haldane JBS: Sex-ratio and unisexual sterility in hybrid animals. J Genet 1922, 12:101-109.

36. Huxley JS: Sexual difference of linkage in Gammarus chereuxi. J Genet 1928, 20:145-156.

37. Rodionov AV: Micro vs. macro: Structural-functional organization of avian micro- and macrochromosomes. Genetika 1996, 32:597-608.

38. Smith J, Bruley CK, Paton IR, Dunn I, Jones CT, Windsor D, Morrice DR, Law AS, Masabanda J, Sazanov A, Waddington D, Fries R, Burt DW: Differences in gene density on chicken macrochromosomes and microchromosomes. Animal Genetics 2000, 31(2):96-103.

39. Myers S, Bottolo L, Freeman C, McVean G, Donnelly P: A fine-scale map of recombination rates and hotspots across the human genome. Science 2005, 310:321-324

40. Shifman S, Bell JT, Copley RR, Taylor MS, Williams RW, Mott R, Flint J: A High-resolution single nucleotide polymorphism genetic map of the mouse genome. PLOS Biol 2006, 4(12):e395.

41. Versteeg $R$, van Schaik BD, van Batenburg MF, Roos M, Monajemi $R$, Caron $\mathrm{H}$, Bussemaker $\mathrm{HJ}$, van Kampen $\mathrm{AH}$ : The human transcriptome map reveals extremes in gene density, intron length, GC content, and repeat pattern for domains of highly and weakly expressed genes. Genome Res 2003, 13(9):1998-2004

42. Spencer CCA, Deloukas P, Hunt S, Mullikin J, Myers S, Silverman B, Donnelly P, Bentley D, McVean G: The Influence of recombination on human genetic diversity. PLoS Genet 2006, 2(9):e148.

doi:10.1186/1471-2164-11-647

Cite this article as: Aslam et al:: A SNP based linkage map of the turkey genome reveals multiple intrachromosomal rearrangements between the Turkey and Chicken genomes. BMC Genomics 2010 11:647.

\section{Submit your next manuscript to BioMed Central and take full advantage of:}

- Convenient online submission

- Thorough peer review

- No space constraints or color figure charges

- Immediate publication on acceptance

- Inclusion in PubMed, CAS, Scopus and Google Scholar

- Research which is freely available for redistribution

Submit your manuscript at www.biomedcentral.com/submit 